\title{
PK/PD Target Attainment With Ceftolozane/ Tazobactam Using Monte Carlo Simulation in Patients With Various Degrees of Renal Function, Including Augmented Renal Clearance and End-Stage Renal Disease
}

\author{
Alan J. Xiao · Luzelena Caro · Myra W. Popejoy · Jennifer A. Huntington • \\ Ravina Kullar
}

Received: November 3, 2016/Published online: December 24, 2016

(c) The Author(s) 2016. This article is published with open access at Springerlink.com

\section{ABSTRACT}

Introduction: Ceftolozane/tazobactam is an antibacterial agent with potent in vitro activity against Gram-negative pathogens, including many extended-spectrum $\beta$-lactamase-producing Enterobacteriaceae and drug-resistant Pseudomonas aeruginosa. Because ceftolozane/tazobactam is primarily excreted renally, appropriate dose adjustments are needed for patients with renal impairment. Monte Carlo simulations were used to determine the probability of pharmacokinetic/ pharmacodynamic target attainment for patients with varying degrees of renal function, including augmented renal clearance (ARC) and end-stage renal disease (ESRD) with hemodialysis.

Methods: Monte Carlo simulations were conducted for 1000 patients with ARC and normal renal function, mild renal impairment,

Enhanced content To view enhanced content for this article go to http://www.medengine.com/Redeem/ BB47F06025963757.

A. J. Xiao · L. Caro · M. W. Popejoy ·

J. A. Huntington · R. Kullar $(\bowtie)$

Merck \& Co., Inc., Kenilworth, NJ, USA

e-mail: ravina.kullar@merck.com moderate renal impairment, or severe renal impairment, and for 5000 patients with ESRD. Simulated dosing regimens were based on approved doses for each renal function category. Attainment targets for ceftolozane were $24.8 \%$ (bacteriostasis), $32.2 \%$ (1-log kill; bactericidal), and $40 \% \quad(2-\log \quad$ kill $)$ $f \mathrm{~T}>$ minimum inhibitory concentration (MIC). The target for tazobactam was to achieve a $20 \%$ $f \mathrm{~T}>$ minimum effective concentration (MEC) at an MEC of $1 \mathrm{mg} / \mathrm{L}$, which was derived from a neutropenic mouse thigh infection model and was confirmed by efficacy data from clinical studies for complicated intraabdominal infections and complicated urinary tract infections.

Results: In patients with ARC or normal renal function, $\geq 91 \%$ achieved bactericidal activity (32.2\% $f \mathrm{~T}>\mathrm{MIC})$ up to an MIC of $4 \mathrm{mg} / \mathrm{L}$ with a 1000-mg ceftolozane dose. In patients with renal impairment (mild, moderate, severe, ESRD), $\geq 93 \%$ achieved bactericidal activity up to an MIC of $8 \mathrm{mg} / \mathrm{L}$. In patients of all renal function categories, the approved dosing regimens of tazobactam achieved $\geq 91 \%$ target attainment against a target of $20 \% f \mathrm{~T}>\mathrm{MEC}$. 
Conclusions: At the approved dosing regimens for ceftolozane/tazobactam, $\geq 91 \%$ of patients in all renal function categories, including ARC (up to $200 \mathrm{~mL} / \mathrm{min}$ ) and ESRD, reached target attainment for bactericidal activity at MICs that correspond to susceptibility breakpoints for Enterobacteriaceae and $P$. aeruginosa.

Keywords: Antibacterial;

Ceftolozane/ tazobactam; Complicated intraabdominal infection; Complicated urinary tract infection; ESRD; Gram-negative pathogens; Monte Carlo simulation; Renal impairment; Target attainment

\section{INTRODUCTION}

Ceftolozane/tazobactam is an antibacterial agent that shows potent in vitro activity against many extended-spectrum $\beta$-lactamase (ESBL)-producing Enterobacteriaceae and drug-resistant Pseudomonas aeruginosa, including multidrug-resistant and extremely drug-resistant isolates [1-3]. Ceftolozane/tazobactam is approved for the treatment of complicated intraabdominal infections (cIAI) when used in combination with metronidazole and for complicated urinary tract infections (cUTI), including pyelonephritis [4].

In pharmacokinetic (PK) studies, ceftolozane/tazobactam demonstrated dose-dependent, linear PK with no clinically relevant drug accumulation with standard every-8-h dosing [4, 5]. Because ceftolozane/tazobactam is eliminated primarily by the kidneys, dosages must be adjusted to account for impaired renal function, specifically for patients with creatinine clearance $(\mathrm{CrCl})$ $\leq 50 \mathrm{~mL} / \mathrm{min}[4,6]$. The primary objective of this analysis was to simulate the probability of
PK/pharmacodynamic (PD) target attainment of ceftolozane/tazobactam in patients with varying degrees of renal impairment, including augmented renal clearance (ARC) and end-stage renal disease (ESRD).

\section{METHODS}

\section{Population PK Model for Simulation}

In the current analysis, PK/PD target attainment for ARC, normal renal function, and mild, moderate, or severe renal impairment was simulated based on a previously developed population $\mathrm{PK}$ model in which $\mathrm{CrCl}$ was a significant covariate [7]. The model was developed with the data from ten clinical studies (eight phase 1 and two phase 2 studies) in healthy subjects with normal renal function, subjects with mildly impaired, moderately impaired, or severely impaired renal function, and patients with cUTI or cIAI [7]. These data included the plasma concentrations of ceftolozane and tazobactam that were collected following intravenous administration of ceftolozane/tazobactam, ceftolozane alone, or tazobactam alone. A two-compartment disposition model with zero-order input and first-order elimination best characterized the plasma concentration-time data for both ceftolozane and tazobactam [7].

PK/PD target attainment for ESRD was simulated based on a previously described population PK model [8]. This model was developed from a PK study in six subjects with ESRD undergoing high-flux hemodialysis (HD) with either Revaclear (Gambro, Stockholm, Sweden) or CT 190G (Baxter Healthcare, McGaw Park, IL, USA) hemodialyzers, and a target adequacy $(\mathrm{Kt} / \mathrm{V})$ of at least 1.2 for a minimum of 3 months before enrollment [6]. 
Subjects were administered a single dose of ceftolozane/tazobactam without HD (i.e., ceftolozane/tazobactam immediately after HD), followed by a washout period with PK sampling, and then a second dose administered $2 \mathrm{~h}$ before a 4-h HD, with intensive PK sampling before and after HD. The collected PK data was then fitted with a nonlinear mixed-effects model with Phoenix NLME software, v.1.2 (Certara L.P. Pharsight, St. Louis, MO, USA). This population PK model is also a two-compartment disposition model to describe the ceftolozane or tazobactam plasma concentration-time data without HD and HD was included as a covariate effect on both clearance and volume of distribution for the central compartment [8].

\section{Monte Carlo Simulation}

Monte Carlo simulations using the population PK models were performed for 1000 patients in each renal function category; 5000 patients were simulated for ESRD. The renal function categories included $\mathrm{ARC}(\mathrm{CrCl},>150$ to $\leq 200 \mathrm{~mL} / \mathrm{min})$, normal renal function $(\mathrm{CrCl}$, $>90$ to $\leq 150 \mathrm{~mL} / \mathrm{min}$ ), mild renal impairment $(\mathrm{CrCl},>50$ to $\leq 90 \mathrm{~mL} / \mathrm{min})$, moderate renal impairment ( $\mathrm{CrCl}, \geq 29$ to $\leq 50 \mathrm{~mL} / \mathrm{min}$ ), severe renal impairment $(\mathrm{CrCl}, \geq 15$ to $<29 \mathrm{~mL} / \mathrm{min})$, and ESRD ( $\mathrm{CrCl},<15 \mathrm{~mL} / \mathrm{min})$. These categories of renal impairment were defined before the US Food and Drug Administration (FDA) updated guidance in 2010 [9], which redefined the cutoff for moderate renal impairment to $30-59 \mathrm{~mL} /$ min, and were retained for consistency of category definitions across trials in the ceftolozane/tazobactam clinical development program. A separate analysis (included in the New Drug Application submission but not shown here) confirmed that definition of renal impairment categories based on the updated guidance would not change the conclusions. In each simulation, except for ESRD, body weight was sampled from a log-normal distribution in the form of $74 \times \exp \left[N\left(0,0.205^{2}\right)\right] \mathrm{kg}$, where $N\left(0,0.205^{2}\right)$ stands for a normal distribution at a mean of 0 with a standard error of 0.205 . This was representative of patients included in the phase 1 and phase 2 clinical trials. In simulations for ESRD, body weight was not relevant because it was not included in the PK model.

Simulated intravenous dosing regimens, administered over $1 \mathrm{~h}$ every $8 \mathrm{~h}$, were based on renal function category and FDA-approved doses [4]: $1.5 \mathrm{~g} \quad(1000 / 500 \mathrm{mg})$ ceftolozane/tazobactam in patients with ARC, normal renal function, or mild renal impairment; $\quad 750 \mathrm{mg} \quad$ (500/250 mg) ceftolozane/tazobactam in patients with moderate renal impairment; $375 \mathrm{mg}$ (250/ $125 \mathrm{mg}$ ) ceftolozane/tazobactam in patients with severe renal impairment; and $750 \mathrm{mg}$ (500/250 mg) ceftolozane/tazobactam loading dose followed by maintenance dose of $150 \mathrm{mg}$ (100/50 mg) ceftolozane/tazobactam over $1 \mathrm{~h}$ every $8 \mathrm{~h}$ for ESRD. Multiple dialysis scenarios were tested for ESRD; we report here the representative weekly scheme of a 4-h HD on Monday, Wednesday, and Friday (i.e., $\mathrm{HD} \rightarrow 2$ days $\rightarrow \mathrm{HD} \rightarrow 2$ days $\rightarrow \mathrm{HD} \rightarrow 3$ days).

A dose was administered immediately following each HD, and the single loading dose was used for the first dose only. Up to 2 cycles (14 days) were simulated for each case, and daily target attainment on day 3 (after the second HD) was the lowest and was reported as a conservative approach.

A finite element method with a time step of $0.001 \mathrm{~h}$ was used to simulate the total concentration-time profiles based on the following mass balance differential equations for the population PK model: 


$$
\begin{gathered}
\mathrm{d} X_{\mathrm{c}} / \mathrm{d} t=R_{t}-\left(\mathrm{Cl}+\mathrm{Q}_{2}\right) / V_{\mathrm{c}} \cdot X_{\mathrm{c}}+\mathrm{Q}_{2} / V_{2} \cdot X_{2} \\
\mathrm{~d} X_{2} / \mathrm{d} t=\mathrm{Q}_{2} / V_{\mathrm{c}} \cdot X_{\mathrm{c}}-\mathrm{Q}_{2} / V_{2} \cdot X_{2}
\end{gathered}
$$

where $X_{\mathrm{c}}$ and $X_{2}$ represent the amount of the drug at time $t$ in the central compartment and peripheral compartment, respectively; $R_{\mathrm{t}}$ represents the infusion rate at time $t ; \mathrm{Cl}$ and $\mathrm{Q}_{2}$ represent the terminal clearance and intercompartmental clearance between the central and peripheral compartments, respectively; and $V_{\mathrm{c}}$ and $V_{2}$ represent the volume of distribution for the central and peripheral compartments, respectively. The population PK model parameter estimates were from the previously published population PK models $[7,8,10]$. To explore the situations in which exposures may be lower in some patients than in typical patients or healthy volunteers at the same dose, however, patients with cIAI were assumed for the simulations. This patient group was selected because it was observed that PK exposure in cIAI patients was lower than in non-cIAI subjects (i.e., cUTI patients or healthy volunteers) [7]. In addition, interindividual variability for the parameter estimates in the PK models was conservatively inflated to have a $50 \%$ coefficient of variation in the log-scale to cover potentially larger variability in real patients.

PK/PD target attainment by minimum inhibitory concentration (MIC) was assessed for ceftolozane by nonclinical PK/PD targets for simulated patients in each renal function category. As with other cephalosporins, the percentage of time with free drug concentration above the MIC ( $\% f \mathrm{~T}>\mathrm{MIC})$ was the PD driver for ceftolozane [11]. The targets for ceftolozane were $24.8 \%$ (bacteriostatic), $32.2 \%$ (for 1-log kill; bactericidal), and 40\% (2-log kill), representing the median $\% f \mathrm{~T}>\mathrm{MIC}$ associated with these levels of activity against
Enterobacteriaceae and $P$. aeruginosa in the neutropenic mouse model [10-13]. The percentage of simulated patients who attained these targets during the dosing interval at steady state for MIC values ranging from 0.03 to $\geq 32 \mathrm{mg} / \mathrm{L}$ was determined for each dosing regimen evaluated within each renal function category. The current Clinical and Laboratory Standards Institute (CLSI) [14] susceptibility breakpoints, which are consistent with the FDA breakpoints, for ceftolozane/tazobactam are $2 \mathrm{mg} / \mathrm{L}$ for Enterobacteriaceae and $4 \mathrm{mg} / \mathrm{L}$ for $P$. aeruginosa.

The target with tazobactam was to achieve $20 \%$ of time above minimum effective concentration (MEC; 20\% $f \mathrm{~T}>\mathrm{MEC}$ ) of $1 \mathrm{mg} / \mathrm{L}$ to effectively inhibit $\beta$-lactamases. The rationale for using the $1 \mathrm{mg} / \mathrm{L}$ tazobactam threshold is based on several in vitro and in vivo studies. In vitro enzyme-binding studies demonstrated that the concentration of $\beta$-lactamase inhibitor required to reduce $\beta$-lactamase enzyme activity by $50 \%\left(\mathrm{IC}_{50}\right)$ is less than $0.3 \mathrm{mg} / \mathrm{L}$ for $>97 \%$ of the $\beta$-lactamases tested $(n=35)$ and for all class A $\beta$-lactamase-producing strains $(n=12)$ [15-17]. Consistent with the $\mathrm{IC}_{50}$ values from these in vitro enzyme-binding experiments, the tazobactam threshold value was determined to be $\leq 1 \mathrm{mg} / \mathrm{L}$ across in vitro dose fractionation and in vivo neutropenic mouse thigh infection PD experiments $[11,18]$. Additionally, $<1 \mathrm{mg} / \mathrm{L}$ was found to be fully effective against all ten clinical strains tested [Escherichia coli $(n=6)$ and Klebsiella pneumoniae $(n=4)]$ in a mouse thigh neutropenic model in which a geometrically averaged $20 \% \quad f \mathrm{~T}>$ threshold of $1 \mathrm{mg} / \mathrm{L}$ tazobactam was observed to be efficacious (data on file). Based on exposure-response relationships determined in the neutropenic murine thigh model for ceftolozane combined with tazobactam, the efficacy target for tazobactam for the $f \mathrm{~T}>$ threshold 
concentration of $1 \mathrm{mg} / \mathrm{L}$ was estimated to be a geometric mean of $19.5 \%$ (mean $25.2 \%$; median 21\%; range 6.6-51.9\%) (internal data). As conceptually illustrated in Fig. 1, based on the typical tazobactam concentration-time profile following administration of $90 \mathrm{mg}$ tazobactam in cIAI patients with normal renal function, the target of $20 \% f \mathrm{~T}>$ MEC of $1 \mathrm{mg} / \mathrm{L}$ is equivalent to the target of $80 \% f \mathrm{~T}>$ threshold of $0.05 \mathrm{mg} /$ $\mathrm{mL}$, which is slightly higher than the target of $70 \% f \mathrm{~T}>$ threshold of $0.05 \mathrm{mg} / \mathrm{L}$ for $2-\log$ kill against isolates with low and moderate $\beta$-lactamase genetic constructs [18], and equivalent to the target of $50 \% f \mathrm{~T}>$ threshold of $0.25 \mathrm{mg} / \mathrm{L}$ for 1-log kill against isolates with high $\beta$-lactamase genetic constructs [18]. In other words, the target of $20 \% f \mathrm{~T}>$ MEC of $1 \mathrm{mg} / \mathrm{L}$, although derived from the neutropenic mouse thigh infection model, is consistent with

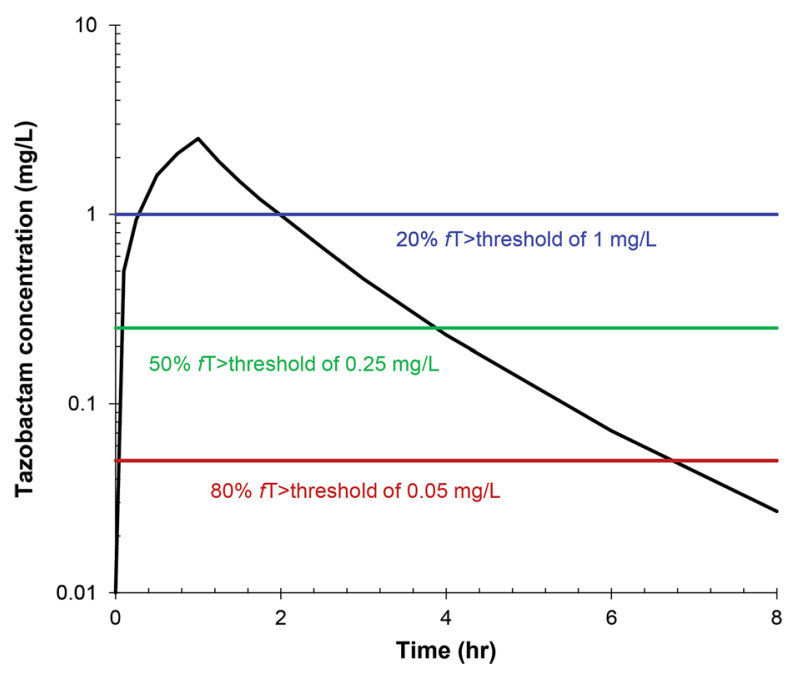

Fig. 1 Typical tazobactam concentration-time profile (after a 1-h infusion of $90 \mathrm{mg}$ tazobactam in patients with cIAI and normal renal function), showing consistency across different target/threshold settings: $20 \% f \Gamma>$ MEC of $1 \mathrm{mg} / \mathrm{L}$ is equivalent to $50 \% f \Gamma>$ threshold of $0.25 \mathrm{mg} /$ $\mathrm{L}$ and $80 \% f \Gamma>$ threshold of $0.05 \mathrm{mg} / \mathrm{L}$. The targets are achieved in $50 \%$ of patients at a dose of $90 \mathrm{mg}$ and can be achieved in $\geq 97 \%$ patients at the approved dose of $500 \mathrm{mg}$ (covering variability). cIAI intraabdominal infection, $f T>M E C$ free-drug time above MEC, $M E C$ minimum effective concentration and even more strict numerically than other observed in vitro and in vivo targets, such that a dose achieving this target will also achieve the other published targets at least for 1-log kill against even the toughest tested $\beta$-lactamase-producing isolates. This is especially true in patients with renal impairment in whom MIC-time profiles display longer half-lives, making it more difficult to achieve a target at a higher concentration threshold than an equivalent target at a lower MIC threshold.

Against non-ESBL-producing pathogens such as $P$. aeruginosa, only target attainment of ceftolozane is relevant and is thus used for dose selection; however, against ESBL-producing pathogens such as Enterobacteriaceae, it is essential to achieve high target attainment for both ceftolozane and tazobactam simultaneously.

In calculations of $\% f \mathrm{~T}>\mathrm{MIC}$ for ceftolozane and $\% f \mathrm{~T}>\mathrm{MEC}$ for tazobactam, unbound fractions $\left(f_{\mathrm{u}}\right)$ of 0.79 and 0.70 were used [10] for the simulated total concentration-time profiles for ceftolozane and tazobactam, respectively.

Statistical analyses and simulations were performed using SAS 9.2 or 9.3 (SAS Institute Inc, NC, USA).

\section{Compliance with Ethics Guidelines}

This article does not contain any new studies with human or animal subjects performed by any of the authors.

\section{Data Availability}

The data sets generated and analyzed during the current study are available from the corresponding author on reasonable request. 


\section{RESULTS}

\section{PK/PD Target Attainment for Ceftolozane}

Systemic exposure to ceftolozane and tazobactam at the approved doses, as reflected by maximum plasma drug concentration $\left(C_{\max }\right)$ and area under the concentration-time curve extrapolated to infinity $\left(\mathrm{AUC}_{0-\infty}\right)$, are presented in Tables 1 and 2, respectively. Only observed $C_{\text {max }}$ and AUC values are reported in the tables; no simulated values. Because no PK data were available from patients with ARC in the clinical trials, no observed values for $C_{\max }$ or AUC are available for those patients.

The most recent surveillance data for ceftolozane/tazobactam (2015) demonstrated that $\mathrm{MIC}_{50 / 90}$ values for isolates from the United States and the European Union, respectively, were $0.5 / 1$ and $0.5 / 16 \mathrm{mg} / \mathrm{L}$ for $P$. aeruginosa and $0.25 / 1$ and $0.25 / 2 \mathrm{mg} / \mathrm{L}$ for Enterobacteriaceae [19; data on file]. Monte Carlo simulation results showed that the percentage of simulated patients achieving $\% f \mathrm{~T}>\mathrm{MIC}$ targets increased as the MIC value or the magnitude of the target decreased. Up to an MIC of $8 \mathrm{mg} / \mathrm{L}, \geq 93 \%$ of patients across all renal function impairment categories (mild, moderate, severe, ESRD) achieved the target for bactericidal activity (i.e., $32.2 \% f \mathrm{~T}>\mathrm{MIC}$ ) (Table 1; Fig. 2a, b).

In the ARC category at the $1.5-\mathrm{g}$ ceftolozane/tazobactam dose, $\geq 91 \%$ of patients achieved $32.2 \% \mathrm{fT}>\mathrm{MIC}$ up to $4 \mathrm{mg} /$ L. Among patients with normal and mild renal impairment, the $32.2 \% f \mathrm{~T}>\mathrm{MIC}$ target was achieved with $1.5 \mathrm{~g}$ ceftolozane/tazobactam in $\geq 96 \%$ of patients at MICs up to $4 \mathrm{mg} / \mathrm{L}$. At the corresponding adjusted doses, $\geq 99 \%$ of patients with moderate to severe renal impairment achieved the $32.2 \% f \mathrm{~T}>\mathrm{MIC}$ targets at MICs up to $4 \mathrm{mg} / \mathrm{L}$. In patients with ESRD, a regimen of 750-mg ceftolozane/tazobactam loading dose followed by $150-\mathrm{mg}$ maintenance dose resulted in $100 \%$ target attainment for up to $40 \%$ $f \mathrm{~T}>\mathrm{MIC}$ targets at MICs up to $4 \mathrm{mg} / \mathrm{L}$ on all days (Table 1).

\section{PK/PD Target Attainment for Tazobactam}

In patients with normal renal function at the 1.5-g ceftolozane/tazobactam dose, the estimated probability of target attainment for tazobactam at the $20 \% f \mathrm{~T}>$ MEC target was $97 \%$ for an MEC of $1 \mathrm{mg} / \mathrm{L}$. Among patients with ARC, $\geq 91 \%$ achieved tazobactam $20 \%$ f $>$ MEC target attainment (Table 2; Fig. 3).

For the mild, moderate, and severe categories of renal impairment, $\geq 99 \%$ of patients achieved the $20 \% f \mathrm{~T}>$ MEC target at the recommended ceftolozane/tazobactam dosing regimen.

For ESRD, the predicted target attainment for tazobactam at the $20 \% f \mathrm{~T}>$ MEC target was $\geq 94 \%$ on all days of the recommended dosing regimen.

\section{DISCUSSION}

Because ceftolozane/tazobactam is renally excreted, renal function is a significant factor influencing PK, with drug clearance decreasing substantially with increasing renal impairment [7]. Appropriate creatinine measurements that can accurately reflect renal function are critical for dose adjustment, especially at the initial doses. If the baseline creatinine measurement is low, dose adjustment may lead to suboptimal exposure and poor treatment outcome. Therefore, supporting clinical markers to confirm actual renal impairment (compared with normal renal function) should be considered before a patient receives a reduced dose. 


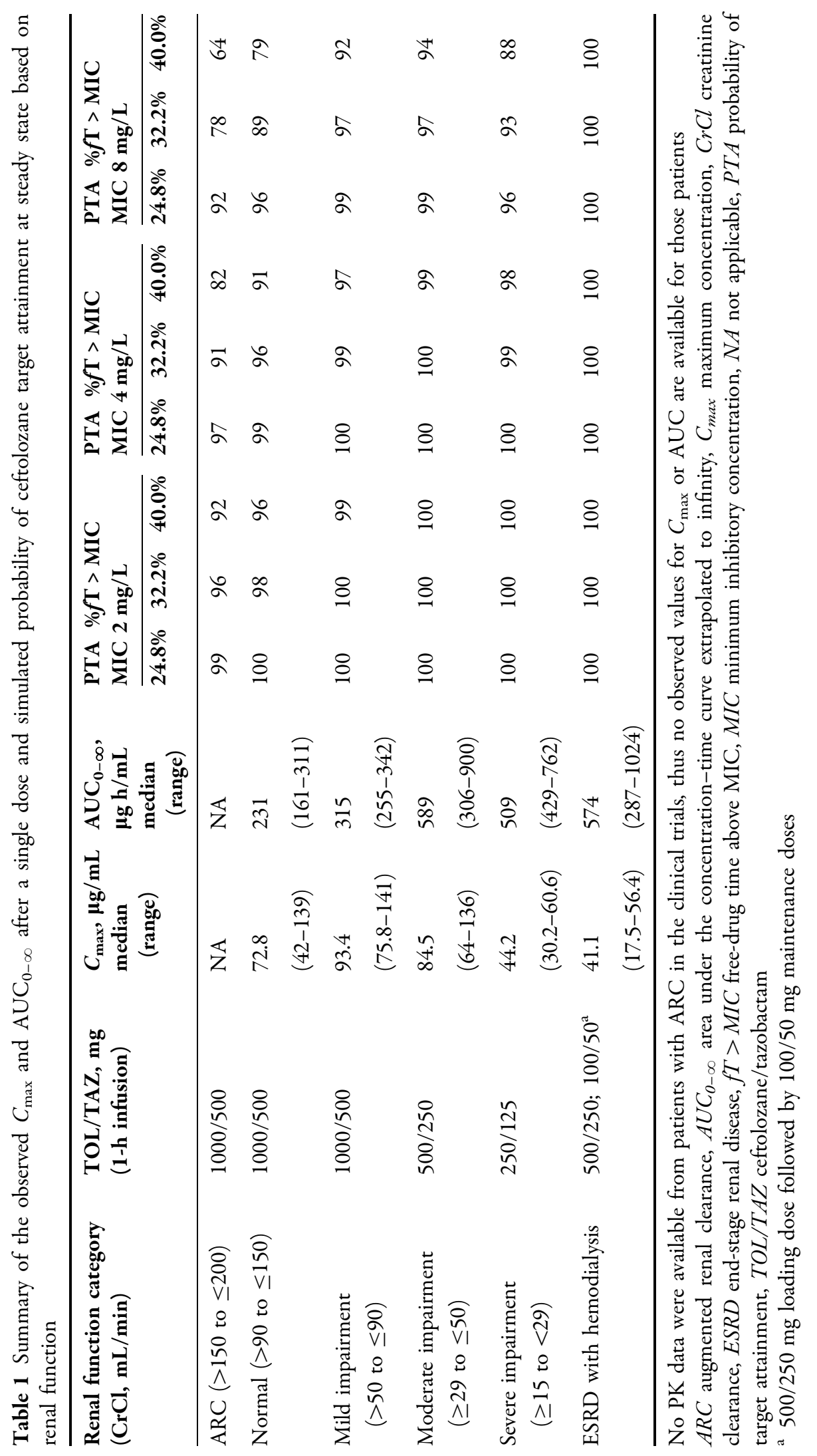


Table 2 Summary of the observed $C_{\max }$ and $\mathrm{AUC}_{0-\infty}$ after a single dose and simulated probability of tazobactam target attainment at steady state based on renal function

\begin{tabular}{lllll}
\hline $\begin{array}{l}\text { Renal function category } \\
(\mathbf{C r C l}, \mathbf{m L} / \mathbf{m i n})\end{array}$ & $\begin{array}{l}\text { TOL/TAZ, mg } \\
(\mathbf{1}-\mathbf{h} \text { infusion) }\end{array}$ & $\begin{array}{l}\boldsymbol{C}_{\text {max }}, \boldsymbol{\mu g} / \mathbf{m L} \\
\text { median }(\mathbf{r a n g e})\end{array}$ & $\begin{array}{l}\mathbf{A U C}_{\mathbf{0}-\infty}, \boldsymbol{\mu g} \mathbf{h} / \mathbf{m L} \\
\text { median }(\mathbf{r a n g e})\end{array}$ & $\begin{array}{l}\text { PTA } \geq 20 \% f \mathbf{T}>\mathbf{M E C}^{\mathbf{b}} \\
\mathbf{M E C}=\mathbf{1} \mathbf{~ m g} / \mathbf{m L}\end{array}$ \\
\hline ARC $(>150$ to $\leq 200)$ & $1000 / 500$ & NA & NA & 91 \\
Normal $(>90$ to $\leq 150)$ & $1000 / 500$ & $17.0(14.7-31.4)$ & $30.1(21.7-40.4)$ & 97 \\
Mild impairment $(>50$ to $\leq 90)$ & $1000 / 500$ & $21.9(18.9-28.3)$ & $34.7(29.1-43.4)$ & 100 \\
$\begin{array}{l}\text { Moderate impairment } \\
\quad(\geq 29 \text { to } \leq 50)\end{array}$ & $500 / 250$ & $27.1(23.3-28.7)$ & $65.9(49.1-91.9)$ & 100 \\
Severe impairment $(\geq 15$ to $<29)$ & $250 / 125$ & $16.3(10.2-18.3)$ & $56.5(35.8-70.9)$ & 99 \\
ESRD with hemodialysis & $500 / 250 ; 100 / 50^{\mathrm{a}}$ & $14.9(7.2-22.9)^{\mathrm{b}}$ & $40.3(23.3-58.6)^{\mathrm{b}}$ & $94^{\mathrm{c}}$ \\
\hline
\end{tabular}

No PK data were available from patients with ARC in the clinical trials, thus, no observed values for $C_{\max }$ or AUC are available for those patients

$A R C$ augmented renal clearance, $A U C_{0-\infty}$ area under the concentration-time curve extrapolated to infinity, $C_{\max }$ maximum concentration, $\mathrm{CrCl}$ creatinine clearance, $E S R D$ end-stage renal disease, $f T>M E C$ free-drug time above MEC, MEC minimum effective concentration, NA not applicable, PTA probability of target attainment, TOL/TAZ ceftolozane/tazobactam

a $500 / 250 \mathrm{mg}$ loading dose followed by $100 / 50 \mathrm{mg}$ maintenance doses

b Measurements taken on hemodialysis and with 500/250 mg dose

${ }^{c}$ Steady state for non-ESRD patients and lowest value on the day immediately after hemodialysis for ESRD/hemodialysis patients

As is the case with other cephalosporins, the efficacy of ceftolozane/tazobactam is best correlated with \% $\mathrm{T}>\mathrm{MIC}[11]$. Using Monte Carlo simulations, we showed that the probability of target attainment in the most conservative case is estimated to be $\geq 91 \%$ for 1-log kill and $\geq 82 \%$ for 2-log kill bactericidal activity in patients with ARC or mild, moderate, or severe renal impairment, and in ESRD patients at the recommended dosing regimens at MICs up to 2 and $4 \mathrm{mg} / \mathrm{L}$, corresponding to the current Enterobacteriaceae and P. aeruginosa breakpoints, respectively. Monte Carlo simulation of tazobactam showed that $\geq 91 \%$ of patients achieved the target of $20 \% f \mathrm{~T}>$ MEC of $1 \mathrm{mg} / \mathrm{L}$ for all renal function categories. Although PK/PD target attainment for tazobactam was not used for dose optimization for the other categories of renal impairment, it was the driver for dose optimization in ESRD patients because the elimination pathway through metabolism (20\% in healthy volunteers with normal renal function) [4,5] became more important than renal clearance in this group of patients.

In general, the achieved high target attainment for the primary targets for both ceftolozane ( $\geq 32.2 \% f \mathrm{~T}>\mathrm{MIC})$ and tazobactam ( $\geq 20 \% f \mathrm{~T}>$ MEC of $1 \mathrm{mg} / \mathrm{L}$ ) at the approved doses was consistent with the high clinical success rate from the phase 3 ASPECT-cUTI and -cIAI trials $[20,21]$, suggesting the validity of the targets.

This study had various limitations. First, although MICs of ceftolozane/tazobactam were determined in the presence of $4 \mathrm{mg} / \mathrm{L}$ tazobactam, as recommended by the CLSI [22], PTA estimates for ceftolozane were based solely on ceftolozane, an approach that has validity for non-ESBL-producing pathogens in patients. 


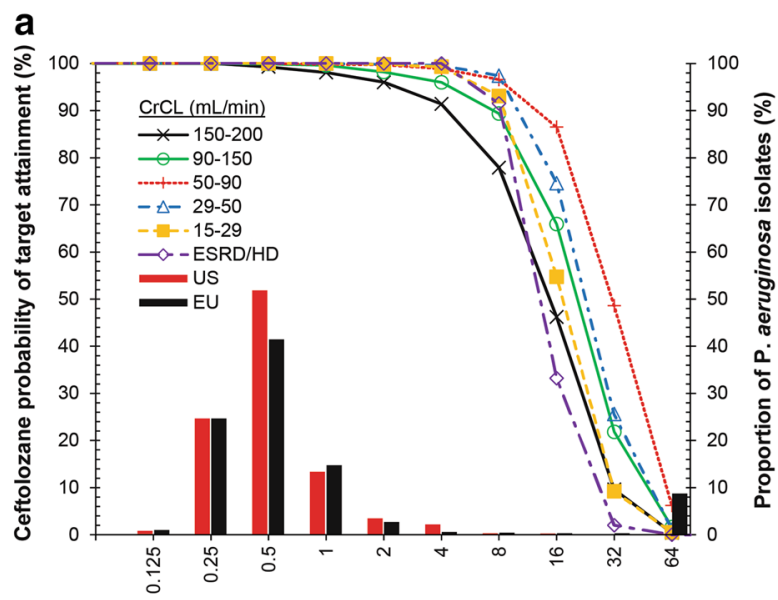

MIC (mg/L)

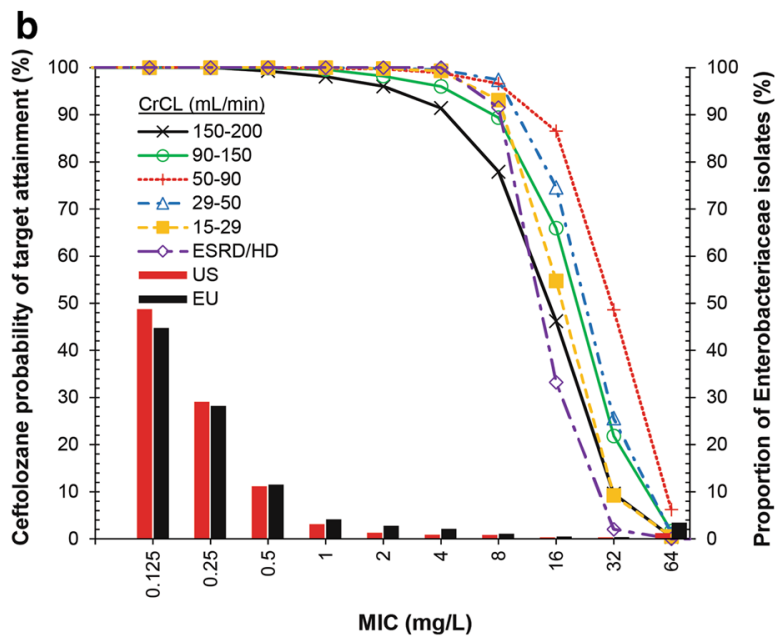

Fig. 2 Simulated ceftolozane PK/PD target attainment [32.2\% $f \Gamma>$ MIC target (1-log kill)] at steady state by renal function group across MIC values following administration of the approved dose regimens. Histograms show MIC distributions for 2015 surveillance isolates [19; data on file]. a $P$. aeruginosa $\left[\mathrm{MIC}_{90}, 1 \mathrm{mg} / \mathrm{L}\right.$ (United States), $16 \mathrm{mg} / \mathrm{L}$ (European Union)]. b Enterobacteriaceae $\left[\mathrm{MIC}_{90}, 1 \mathrm{mg} / \mathrm{L}\right.$ (United States), $2 \mathrm{mg} / \mathrm{L}$ (European Union)]. $\mathrm{CrCl}$ creatinine clearance, ESRD end-stage renal disease, $H D$ hemodialysis, $M I C$ minimum inhibitory concentration, $P D$ pharmacodynamics, $P K$ pharmacokinetics

For ESBL-producing pathogens, published data support tazobactam as an inhibitor of $\beta$-lactamase activity and indicate that the PD driver for tazobactam is the percentage of time above a threshold concentration (\% $f \mathrm{~T}>$ threshold) [23]. Given that our data

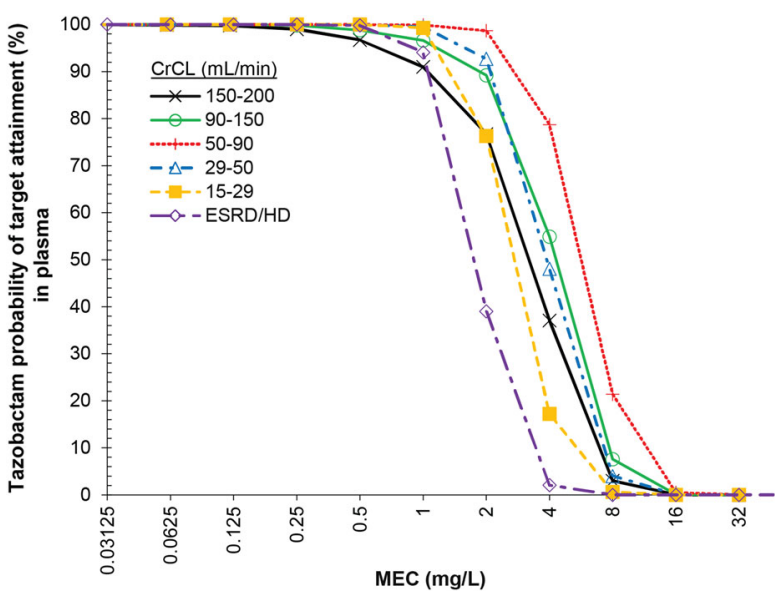

Fig. 3 Simulated tazobactam PK/PD target attainment $(20 \% f \Gamma>M E C)$ at steady state by renal function group across MEC values following administration of the approved dose regimens. $\mathrm{CrCl}$ creatinine clearance, ESRD end-stage renal disease, $f T>M E C$ free-drug time above MEC, $H D$ hemodialysis, $M E C$ minimum effective concentration, $P D$ pharmacodynamics, $P K$ pharmacokinetics

suggest that the highest tazobactam threshold was $1 \mathrm{mg} / \mathrm{L}$ against ESBL-producing pathogens, under the condition of high attainment for this target, PTA calculations using ceftolozane alone appear to be a practical and reasonable approach. PTA calculations based on the combination of ceftolozane and tazobactam are mechanistically interesting, but the methodology on the optimal way to model two components (a cephalosporin and a $\beta$-lactamase inhibitor) simultaneously is still under discussion, and several potential approaches have been proposed [24-27]. Nevertheless, the individual exposure of ceftolozane (\% $\mathrm{T}>\mathrm{MIC})$ and tazobactam (\% $\mathrm{T}>\mathrm{MEC}$ ) in patients with normal function is high at the 1.5-g dose and was confirmed to be efficacious in clinical trials for cUTIs and cIAIs against both non-ESBL-producing and ESBL-producing pathogens [20, 21]. Second, this study was limited by the lack of clinical data to support the findings in ARC, severe renal impairment, and ESRD. Recent case studies, however, have reported successful clinical 
outcomes in patients with more severe renal impairment $[28,29]$. Third, this study was based on population PK models and simulations with characteristics from patients not critically ill, though still infected, or from patients with ESRD who were otherwise healthy. In contrast, many critically ill patients have lower drug clearances, larger volumes of distribution, and, consequently, longer terminal half-lives than healthy persons. These factors are to be confirmed by the ongoing study in critically ill patients (ClinicalTrials.gov, NCT02387372). Finally, this study does not include the case for patients with ARC higher than $200 \mathrm{~mL} / \mathrm{min}$ or the case for tissue infection in which penetration of the drug into the infected tissue site might be low (for example, penetration into lung tissue in patients with pneumonia). In both cases, a higher dose might be necessary. Indeed, a higher dose of $3 \mathrm{~g}$ ceftolozane/tazobactam has been well tolerated in PK studies [10, 30] and is being evaluated in a phase 3 trial in patients with ventilated nosocomial pneumonia (ClinicalTrials.gov, NCT02070757).

\section{CONCLUSIONS}

This analysis confirms that the approved dosing regimens for ceftolozane/tazobactam in patients with mild, moderate, or severe renal impairment and in patients with ESRD are sufficient to achieve high target attainment for bactericidal activity at all the approved breakpoints.

\section{ACKNOWLEDGEMENTS}

Sponsorship for this simulation study and article processing funds were provided by Merck \& Co., Inc., Kenilworth, NJ, USA. All authors had full access to all the data in this study and take complete responsibility for the integrity of the data and the accuracy of the data analysis. Editorial assistance in the preparation of this manuscript was provided by Sally Mitchell, PhD, and Meher Dustoor, PhD, of ApotheCom, Yardley, PA, USA. This assistance was funded by Merck \& Co., Inc., Kenilworth, NJ, USA. All named authors meet the International Committee of Medical Journal Editors (ICMJE) criteria for authorship for this manuscript, take responsibility for the integrity of the work as a whole, and have given final approval to the version to be published.

Disclosures. Alan J. Xiao was an employee of Merck \& Co., Inc., Kenilworth, NJ, USA, at the time the data used in these analyses were generated; he is now an employee of Novartis. Luzelena Caro is an employee of Merck \& Co., Inc., Kenilworth, NJ, USA. Myra W. Popejoy is an employee of Merck \& Co., Inc., Kenilworth, NJ, USA. Jennifer A. Huntington is an employee of Merck \& Co., Inc., Kenilworth, NJ, USA. Ravina Kullar is an employee of Merck \& Co., Inc., Kenilworth, NJ, USA.

Compliance with Ethics Guidelines. This article does not contain any new studies with human or animal subjects performed by any of the authors.

Data Availability. The data sets generated and analyzed during the current study are available from the corresponding author on reasonable request.

Open Access. This article is distributed under the terms of the Creative Commons Attribution-NonCommercial 4.0 International License (http://creativecommons.org/licenses/ by-nc/4.0/), which permits any noncommercial 
use, distribution, and reproduction in any medium, provided you give appropriate credit to the original author(s) and the source, provide a link to the Creative Commons license, and indicate if changes were made.

\section{REFERENCES}

1. Zhanel GG, Chung $\mathrm{P}$, Adam $\mathrm{H}$, et al. Ceftolozane/tazobactam: a novel cephalosporin/beta-lactamase inhibitor combination with activity against multidrug-resistant gram-negative bacilli. Drugs. 2014;74:31-51.

2. Farrell DJ, Sader HS, Flamm RK, Jones RN. Ceftolozane/tazobactam activity tested against Gram-negative bacterial isolates from hospitalised patients with pneumonia in US and European medical centres (2012). Int J Antimicrob Agents. 2014;43:533-9.

3. Farrell DJ, Flamm RK, Sader HS, Jones RN. Antimicrobial activity of ceftolozane-tazobactam tested against Enterobacteriaceae and Pseudomonas aeruginosa with various resistance patterns isolated in U.S. hospitals (2011-2012). Antimicrob Agents Chemother. 2013;57:6305-10.

4. Zerbaxa (ceftolozane and tazobactam) [prescribing information]. Whitehouse Station: Merck Sharp \& Dohme, 2015.

5. Miller B, Hershberger E, Benziger D, Trinh M, Friedland I. Pharmacokinetics and safety of intravenous ceftolozane-tazobactam in healthy adult subjects following single and multiple ascending doses. Antimicrob Agents Chemother. 2012;56:3086-91.

6. Wooley M, Miller B, Krishna G, Hershberger E, Chandorkar G. Impact of renal function on the pharmacokinetics and safety of ceftolozane-tazobactam. Antimicrob Agents Chemother. 2014;58:2249-55.

7. Chandorkar G, Xiao A, Mouksassi MS, Hershberger E, Krishna G. Population pharmacokinetics of ceftolozane/tazobactam in healthy volunteers, subjects with varying degrees of renal function and patients with bacterial infections. J Clin Pharmacol. 2015;55:230-9.

8. Xiao A, Chandorkar G, Krishna G, Hershberger E. Ceftolozane/tazobactam $(\mathrm{C} / \mathrm{T})$ dose optimization in patients with end stage renal disease (ESRD) requiring hemodialysis (HD) using population pharmacokinetics (pPK) and Monte Carlo simulations (MCS). Presented at: MAD-ID 18th Annual Congress; May 7-9, 2015; Orlando.

9. US Department of Health and Human Services. Guidance for industry: pharmacokinetics in patients with impaired renal function-study design, data analysis, and impact on dosing and labeling (March 2010). Report No. 91. http://www. fda.gov/downloads/Drugs/.../Guidances/UCM2049 59.pdf. Accessed October 25, 2016.

10. Xiao AJ, Miller BW, Huntington JA, Nicolau DP. Ceftolozane/tazobactam pharmacokinetic/ pharmacodynamic derived dose justification for phase 3 studies in patients with nosocomial pneumonia. J Clin Pharmacol. 2015;56:56-66.

11. Craig WA, Andes DR. In vivo activities of ceftolozane, a new cephalosporin, with and without tazobactam against Pseudomonas aeruginosa and Enterobacteriaceae, including strains with extended-spectrum beta-lactamases, in the thighs of neutropenic mice. Antimicrob Agents Chemother. 2013;57:1577-82.

12. Drusano GL. Antimicrobial pharmacodynamics: critical interactions of 'bug and drug'. Nat Rev Microbiol. 2004;2:289-300.

13. Lepak AJ, Reda A, Marchillo K, Van Hecker J, Craig WA, Andes D. Impact of MIC range for Pseudomonas aeruginosa and Streptococcus pneumoniae on the ceftolozane in vivo pharmacokinetic/ pharmacodynamic target. Antimicrob Agents Chemother. 2014;58:6311-4.

14. Clinical and Laboratory Standards Institute. M100-S26 performance standards for antimicrobial susceptibility testing: twenty-sixth edition. Wayne: CLSI; 2016.

15. Payne DJ, Cramp R, Winstanley DJ, Knowles DJ. Comparative activities of clavulanic acid, sulbactam, and tazobactam against clinically important beta-lactamases. Antimicrob Agents Chemother. 1994;38:767-72.

16. Bush K, Macalintal C, Rasmussen BA, Lee VJ, Yang Y. Kinetic interactions of tazobactam with beta-lactamases from all major structural classes. Antimicrob Agents Chemother. 1993;37:851-8.

17. Naumovski L, Quinn JP, Miyashiro D, et al. Outbreak of ceftazidime resistance due to a novel extended-spectrum beta-lactamase in isolates from cancer patients. Antimicrob Agents Chemother. 1992;36:1991-6.

18. Vanscoy B, Mendes RE, Nicasio AM, et al. Pharmacokinetics-pharmacodynamics of 
tazobactam in combination with ceftolozane in an in vitro infection model. Antimicrob Agents Chemother. 2013;57:2809-14.

19. Huband MD, Flamm RK, Jones RN, Sader HS, Farrell DJ. In vitro activity of ceftolozane/tazobactam against pseudomonas aeruginosa and enterobacteriaceae isolates collected from medical centers in the USA (2015) 2015. Presented at: American Society for Microbiology (ASM) Microbe 2016; June 16-20, 2016; Boston, MA. Poster 431.

20. Solomkin J, Hershberger E, Miller B, et al. Ceftolozane/tazobactam plus metronidazole for complicated intra-abdominal infections in an era of multidrug resistance: results from a randomized, double-blind, phase 3 trial (ASPECT-cIAI). Clin Infect Dis. 2015;60:1462-71.

21. Wagenlehner FM, Umeh O, Steenbergen J, Yuan G, Darouiche RO. Ceftolozane-tazobactam compared with levofloxacin in the treatment of complicated urinary-tract infections, including pyelonephritis: a randomised, double-blind, phase 3 trial (ASPECT-cUTI). Lancet. 2015;385:1949-56.

22. Clinical Laboratory Standards Institute. Methods for dilution antimicrobial susceptibility tests for bacteria that grow aerobically: report no. M07-A09; ninth edition. Wayne: CLSI, 2012.

23. Vanscoy B, Mendes RE, Castanheira $M$, et al. Relationship between ceftolozane-tazobactam exposure and drug resistance amplification in a hollow-fiber infection model. Antimicrob Agents Chemother. 2013;57:4134-8.

24. Bhagunde P, Chang KT, Hirsch EB, Ledesma KR, Nikolaou M, Tam VH. Novel modeling framework to guide design of optimal dosing strategies for beta-lactamase inhibitors. Antimicrob Agents Chemother. 2012;56:2237-40.

25. Rubino C, Bhavnani SM, Steenbergen JN, Krishna G, Ambrose PG. Pharmacokinetic-pharmacodynamic (PK-PD) target attainment analyses supporting the selection of in vitro susceptibility test interpretive criteria for ceftolozane/tazobactam (TOL/TAZ) against Enterobacteriaceae. Presented at: 54th Annual Interscience Conference on Antimicrobial Agents and Chemotherapy; September 5-9, 2014; Washington, DC. Poster A-1347.

26. Melchers MJ, Mavridou E, Seyedmousavi S, van Mil AC, Lagarde C, Mouton JW. Plasma and ELF pharmacokinetics of ceftolozane and tazobactam alone and in combination in mice. Antimicrob Agents Chemother. 2015;59:3373-6.

27. European Medicines Agency. Guidelines on the use of pharmacokinetics and pharmacodynamics in the development of antimicrobial medicinal products. http://www.ema.europa.eu/docs/en_GB/document library/Scientific_guideline/2016/07/WC500210982. pdf. Accessed 25 Oct 2016.

28. Bremmer DN, Nicolau DP, Burcham P, Chunduri A, Shidham G, Bauer KA. Ceftolozane/tazobactam pharmacokinetics in a critically ill adult receiving continuous renal replacement therapy. Pharmacotherapy. 2016;36:e30-3.

29. Patel UC, Nicolau DP, Sabzwari RK. Successful treatment of multi-drug resistant Pseudomonas aeruginosa bacteremia with the recommended renally adjusted ceftolozane/tazobactam regimen. Infect Dis Ther. 2016;5:73-9.

30. Miller B, Chandorkar G, Umeh O, Friedland I, Hershberger E. Safety and pharmacokinetics (PK) of intravenous (IV) ceftolozane/tazobactam (C/T) every 8 hours $(18 \mathrm{~h})$ and cumulative fraction of response (CFR) in plasma and epithelial lining fluid (ELF) in a simulated ventilator-associated pneumonia (VAP) population. Presented at: 52nd Interscience Conference on Antimicrobial Agents and Chemotherapy, September 9-12, 2012; San Francisco, CA. 